Comparison of Umbilical Serum Copeptin Relative to Erythropoietin and S100B as Asphyxia Biomarkers at Birth

\title{
Summanen, Milla
}

2017

Summanen , M , Seikku , L , Rahkonen , P , Stefanovic , V , Teramo , K, Andersson , S , Kaila , K \& Rahkonen , L 2017 , ' Comparison of Umbilical Serum Copeptin Relative to Erythropoietin and S100B as Asphyxia Biomarkers at Birth ', Neonatology , vol. 112 , no. 1 , pp. 60-66 . https://doi.org/10.1159/000456063

http://hdl.handle.net/10138/310867

https://doi.org/10.1159/000456063

acceptedVersion

Downloaded from Helda, University of Helsinki institutional repository.

This is an electronic reprint of the original article.

This reprint may differ from the original in pagination and typographic detail.

Please cite the original version. 
1 Comparison of umbilical serum copeptin relative to erythropoietin and S100B as asphyxia

2 biomarkers at birth

3

4 Milla Summanen ${ }^{\text {a,b }}$, Laura Seikku ${ }^{\text {c }}$, Petri Rahkonen ${ }^{\text {c,d }}$, Vedran Stefanovic ${ }^{c}$, Kari Teramo ${ }^{c}$, Sture

5 Andersson $^{\mathrm{d}}$, Kai Kaila ${ }^{\mathrm{a}, \mathrm{b}}$, Leena Rahkonen ${ }^{\mathrm{c}}$

6

7 a Department of Biosciences, University of Helsinki, Helsinki, Finland; ${ }^{\mathrm{b}}$ Neuroscience Center,

8 University of Helsinki, Helsinki, Finland; ${ }^{c}$ Department of Obstetrics and Gynecology, University of

9 Helsinki and Helsinki University Hospital, Helsinki, Finland; and ${ }^{\mathrm{d}}$ Children’s Hospital, Helsinki

10 University Hospital, Helsinki, Finland

11

12 Short title: Birth asphyxia biomarkers

13

14 Keywords: neonate, birth asphyxia, arginine vasopressin (AVP), copeptin, erythropoietin, S100B 15

30 Corresponding author: Kai Kaila, Department of Biosciences, Viikinkaari 1, 00790 Helsinki,

31 Finland, tel. +358 40 7256 759, [kai.kaila@ helsinki.fi]. 
Abstract

34 Background: Birth asphyxia, estimated to account for a million neonatal deaths annually, can cause 35 a wide variety of neurodevelopmental impairments. There is a need to develop new, swift methods to identify those neonates who would benefit from neuroprotective treatments such as hypothermia.

37 Objectives: To examine the utility of cord serum copeptin, a stable byproduct of arginine vasopressin 38 release, as a biomarker of birth asphyxia based on a comparison with two biomarkers of hypoxia and 39 brain trauma, erythropoietin and S100B.

40 Methods: The study population consisted of 140 singleton, term neonates; 113 controls and 27 with 41 birth asphyxia (two/three criteria met: umbilical artery $\mathrm{pH}<7.10$, base excess $<-12 \mathrm{mmol} / 1$, and 5 minute Apgar score <7). All deliveries were planned vaginal, but 51 neonates were born by emergency cesarean section. Copeptin, S100B, and erythropoietin levels in umbilical artery samples were measured by immunoassays.

45 Results: Copeptin correlated in the entire study population more strongly with umbilical artery base excess than S100B and erythropoietin, and only copeptin correlated with arterial $\mathrm{pH}$. Furthermore, only copeptin levels were significantly higher in cases of birth asphyxia, and in vaginally born neonates they were found to increase as a function of labor duration. Copeptin was elevated in neonates born via vacuum extraction, whereas erythropoietin levels showed a slight increase after emergency cesarean section.

51 Conclusions: In this study population, S100B and erythropoietin were not valid biomarkers of birth 52 asphyxia. In contrast, our work suggests that copeptin has high potential to become a routinely used biomarker for acute birth asphyxia and neonatal distress. 
Birth asphyxia is a severe clinical problem globally, which has been estimated to account for a million of neonatal deaths annually [1]. The neurodevelopmental impairments in individuals who develop neonatal hypoxic-ischemic encephalopathy (HIE) range from minor cognitive problems and sensorimotor defects to cerebral palsy [1, 2]. Methods for identifying those neonates who would benefit from neuroprotective treatments such as therapeutic hypothermia is a major challenge because of the restricted time window of only a few hours for decision making. The increasing risk of HIE associated with enhanced acidemia has been well established. The Apgar scores at 5 minutes are known to correlate with the risk of neurological disability [3]. However, the sensitivity and specificity of arterial cord blood $\mathrm{pH}$ values and Apgar scores with regard to outcome following HIE are low [2]. Thus, reliable biomarkers for predicting outcome after birth asphyxia are urgently needed.

A number of studies on blood-borne protein biomarkers in neonates have been published in recent years [4-6]. Among these, erythropoietin (EPO) is a biomarker of chronic hypoxia [7], and high levels of umbilical plasma EPO at birth are associated with an increased risk for adverse outcome [8]. S100B is considered as a biomarker of brain cell damage [9], and its levels are known to rise at the early phase of acute asphyxia [6]. In the present study, we examine the utility of cord serum copeptin, a byproduct of arginine vasopressin (AVP) release, as a biomarker of birth asphyxia based on a comparison with EPO and S100B. The rationale of this approach lies in the fact that a massive surge of AVP release takes place during normal vaginal birth in response to activation of the fetal hypothalamic-pituitary-adrenal (HPA) axis, which is further accentuated by various kinds of stress factors such as infections, intra-uterine growth restriction, and acidosis/birth asphyxia $[4,5,10,11]$. In contrast to AVP, copeptin is biochemically stable with a much longer half-life, and it is released in an equimolar ratio to AVP, making it an ideal surrogate for AVP measurements $[11,12]$. One of the practical advantages of copeptin is that it is widely used as a biomarker of various pathophysiological states like lower respiratory tract infections, septic shock and stroke in emergency departments [13].

\section{Methods}

83 Study design and patients 
84 The serum samples for this retrospective study were collected in the Department of Obstetrics and 85 Gynecology, Helsinki University Hospital, Finland between May 2012 and April 2013. The study, approved by the local Ethics Committee of the Department of Obstetrics and Gynecology, Helsinki University Hospital, Finland (105/13/13/03/2012), consisted of 151 singleton births at or beyond $37+0$ gestational weeks. Umbilical artery blood samples were collected immediately after birth from 72 neonates with suspected asphyxia, based on a 1-minute Apgar score <4. The 1-minute Apgar score was used only for patient recruitment, to allow midwives to identify neonates suspected of having suffered from birth asphyxia. Umbilical artery cord blood samples from 79 control neonates with a 1-minute Apgar score $\geq 4$ were collected during the five-day work week. Pregnancies complicated by maternal type 1 diabetes $(n=1)$, preeclampsia $(n=3)$, fetal growth restriction $(n=4)$ and $R h$ immunization $(n=1)$ were excluded. In addition, one neonate was excluded because of chromosomal anomaly and one had mitochondrial disease, leaving a total of 140 neonates for the final analysis. The neonates were divided into two groups, asphyxia and control, for data analysis. The neonate was considered to fulfill the diagnostic criteria for birth asphyxia if two of three criteria were met: umbilical artery $\mathrm{pH}<7.1$, umbilical artery base excess $<-12 \mathrm{mmol} / \mathrm{l}$, and a 5-minute Apgar score $<7$ $[14,15]$.

Gestational age was defined by fetal crown-rump length measurement at the first trimester ultrasound screening. Deliveries were all planned vaginal. Indications for emergency cesarean sections (ECS) were: fetal distress $(n=29)$, labor dystocia $(n=10)$, prolonged second stage of delivery $(n=2)$, chorionamnionitis $(n=1)$, fetal malpresentation $(n=3)$, unsuccessful vacuum extraction $(n=4)$, and umbilical cord prolapse $(n=2)$. Data on maternal pregnancy characteristics and short-term perinatal outcome were collected from the hospital charts (table 1). Birth weight z-score was defined according to the Finnish population standardized for sex and gestational age [16].

Blood samples from the umbilical artery were used for measurements of $\mathrm{pH}$, base excess ( $\mathrm{BE}$ ), $\mathrm{pO}_{2}$ and $\mathrm{pCO}_{2}$ (Radiometer ABL800 Flex blood gas analyzer, Copenhagen, Denmark). Serum samples from the umbilical artery were used for biomarker measurements.

\section{Copeptin measurements}

114 We used a sandwich enzyme-linked immunosorbent assay (ELISA, methodological details in the 115 Supplementary material). The inter-assay variability was $5.8 \%$, which was estimated by calculating 4 
116 the CV from eight duplicate samples run on two plates on two separate days. Fifteen serum samples 117 were analyzed with both the ELISA and the BRAHMS copeptin Kryptor assay used in previous 118 publications [4, 5], and a highly significant linear correlation (Pearson $r=0.9793, p<0.0001$ ) was 119 found, covering the full range of values obtained using the Kryptor, 6.0 to $4637 \mathrm{pmol} / \mathrm{l}$ (median 381.2 $120 \mathrm{pmol} / \mathrm{l})$. This excellent linear correlation, with an $\mathrm{R}^{2}$ of 0.9591 indicating a congruence of $96 \%$ 121 between the two methods, was used to convert the copeptin concentrations obtained with the ELISA 122 to Kryptor concentrations. This allows for a direct comparison of our data with the already published 123 copeptin results in neonates.

\section{S100B measurements}

126 Serum S100B was measured with electrochemiluminometric immunoassay using Modular e170 127 analyzer (Roche Diagnostics). The detection range for the S100B assay is $0.005 \mu \mathrm{g} / 1$ and functional 128 sensitivity less than $0.02 \mu \mathrm{g} / \mathrm{l}$. The intra-assay coefficient of variation (CV) was less than $2.1 \%$ and 129 inter-assay variation better than $6.4 \%$.

\section{EPO measurements}

132 EPO was measured using a solid-phase chemiluminescent enzyme immunometric assay Immulite 1332000 XPI analyser (Siemens Healthcare Diagnostics). The intra-assay CV was 3.6-6.8 \%, while the 134 total CV was 6.4-10.3\%. Detection limit was $1.0 \mathrm{IU} / \mathrm{l}$, and functional sensitivity (CV $20 \%$ ) was 1.5 $135 \mathrm{IU} / 1$.

\section{Statistical analysis}

138 All three biomarkers were measured from each of the 140 samples included in this study. The 139 following statistical analyses were performed with GraphPad Prism 6 or SPSS 22. Prior to calculating 140 correlations and significances, statistical outliers $(\mathrm{p}<0.01)$ for copeptin and S100B ( $\mathrm{n}=1$ for each) 141 were excluded from the study population. Correlations between the $\mathrm{pH}, \mathrm{BE}, \mathrm{S} 100 \mathrm{~B}, \mathrm{EPO}$ and 142 copeptin values from all remaining samples were calculated with the Spearman correlation 143 coefficient. Copeptin, S100B and EPO values between the study groups were compared using the 144 Mann-Whitney U test or the Kruskall-Wallis test, and a receiver operating characteristic (ROC)-curve 145 was drawn to determine the diagnostic accuracy of copeptin to birth asphyxia. The Chi-squared test 146 or Fisher's exact test were used to determine significant differences in the study population. 


\section{$148 \quad$ Results}

Maternal and fetal/delivery characteristics

150 The maternal and fetal/delivery characteristics are shown in table 1 . There were no statistically 151 significant differences in any of the maternal or delivery characteristics between the two study groups.

Dependence of biomarker levels on umbilical artery $\mathrm{pH}$ and $\mathrm{BE}$

154 The neonates' median $\mathrm{pH}, \mathrm{BE}, \mathrm{pO}_{2}$ and $\mathrm{pCO}_{2}$ values, which are routinely measured from umbilical 155 arterial cord blood at birth, are shown in table 1. The dependence of the three biomarkers on the blood acid-base parameters are given in figure 1. Only copeptin levels showed a significant correlation with umbilical artery $\mathrm{pH}(\mathrm{r}=-0.6219, \mathrm{p}<0.0001$, fig. 1a). All three biomarkers correlated with umbilical artery BE (fig. 1d-f), with copeptin showing by far the highest correlation coefficient $(r=-0.6372, p$ $159<0.0001)$.

\section{Biomarker levels and asphyxia}

162 Twenty-seven neonates in the study population belonged to the birth asphyxia group (see Methods 163 for present criteria). Copeptin levels were significantly higher among the neonates in the birth 164 asphyxia group compared to unaffected controls (mean $2450 \mathrm{pmol} / 1 \mathrm{vs} 1226 \mathrm{pmol} / \mathrm{l}$, p < 0.0001, fig. 165 2c), whereas no differences in S100B (fig. 2a) or EPO (fig. 2b) levels were found. ROC-curve analysis 166 showed that copeptin concentrations discriminated with moderate accuracy between asphyxia, as 167 defined in this study, and controls: the area under the curve was 0.76 (95\%-CI 0.69-0.86, fig. 2d). A cut-off of $1522 \mathrm{pmol} / \mathrm{l}$ had a sensitivity of $77 \%$ and a specificity of $70 \%$.

Biomarker levels and delivery mode

171 All 140 deliveries were planned vaginal, but 51 neonates were ultimately born by ECS. Of the 89

172 vaginal deliveries, 33 were assisted with vacuum extraction. There were no differences in the delivery 173 mode between the asphyxia and control study groups (table 1). Copeptin levels were higher among 174 the neonates born via vacuum extraction as compared to ECS (mean $2021 \mathrm{pmol} / \mathrm{l}$ vs $1190 \mathrm{pmol} / \mathrm{l}, \mathrm{p}$ $175=0.003$, fig. 3a) or normal vaginal delivery, although the latter difference did not reach statistical 176 significance (mean $2021 \mathrm{pmol} / \mathrm{l}$ vs $1362 \mathrm{pmol} / \mathrm{l}, \mathrm{p}=0.0522$, fig. 3a). No differences in S100B levels 177 were found between the groups (fig. 3b), whereas EPO levels were higher among neonates born via 
178 ECS compared to normal vaginal delivery or vacuum extraction (means $713.7 \mathrm{U} / 1,78.41 \mathrm{U} / 1$, and $17961.15 \mathrm{U} / \mathrm{l}$, respectively; $\mathrm{p}=0.0002$ and $\mathrm{p}=0.001$, fig. $3 \mathrm{c}$ ), which might reflect prenatal conditions 180 [7].

Biomarker levels in relation to other variables

183 S100B levels were higher in male neonates $(\mathrm{p}=0.0378)$, but copeptin and EPO levels did not differ 184 based on the sex of the neonate. All biomarkers showed a correlation with the 5-minute Apgar score, 185 and copeptin and S100B correlated also with the 10-minute Apgar score (table 2). Copeptin and 186 S100B levels did not correlate with gestational age at birth, whereas EPO levels did $(r=0.4513, p<$ 187 0.0001). Only copeptin levels correlated significantly with birth weight $(\mathrm{r}=-0.1713, \mathrm{p}=0.0438)$. 188 Among the 89 neonates born vaginally, copeptin levels increased as a function of the total duration 189 of labor $(\mathrm{r}=0.3267, \mathrm{p}=0.0019)$ and the duration of the second stage of labor $(\mathrm{r}=0.2787, \mathrm{p}=0.0086$; 190 table 2). EPO and S100B levels did not correlate with either of these variables.

\section{Discussion}

193 A wide spectrum of adaptive processes in respiratory, cardiovascular, and metabolic functions are 194 triggered at birth [4, 10]. Changes in biomarker concentrations during birth reflect, at least in part, 195 physiological fetal adaptive reactions, such as enhanced activation of the HPA-axis (see below), in 196 response to normal or complicated delivery. Distinct biomarkers have different profiles during fetal 197 and neonatal asphyxia $[4,7,18]$. The S100B level in plasma has been shown to rise at the early phase 198 of acute asphyxia [6, 18] and significantly higher S100B levels have been reported in asphyxiated 199 term neonates with intraventricular hemorrhage (IVH) or with HIE, compared to asphyxiated 200 neonates without IVH/HIE or to apparently healthy neonates [19]. EPO is a biomarker of chronic 201 hypoxia, and increased levels can be detected in fetal plasma and amniotic fluid in various 202 pathological pregnancies [7]. An association of high EPO levels during pregnancy and adverse acute 203 neonatal outcome, such as decreased umbilical cord $\mathrm{pH}, \mathrm{pO}_{2}$ and $\mathrm{BE}$, and increased intensive care 204 unit admission, has also been reported [7, 20]. Furthermore, high levels of umbilical plasma EPO at 205 birth are associated with an increased risk for death or abnormal neurological outcome at two years 206 of age [8]. 
208 There are many a priori reasons why copeptin might turn out to be a highly useful biomarker of birth 209 asphyxia, and a number of previous observations point in this direction [4, 5, 21]. During birth, the 210 HPA axis shows massive activation, which results in the release of AVP, and this response is further 211 enhanced by various types of stressors. Since AVP is highly unstable with a short half-life of 4-20 212 minutes [22], assays of this hypothalamic hormone itself are not suitable for clinical use. However, 213 AVP is derived from a larger precursor peptide which contains copeptin, a stable C-terminal fragment 214 with 39 amino acids. Copeptin is released in an equimolar ratio to AVP [23]. In line with the 215 underlying HPA-based mechanisms, copeptin levels in cord blood increase in different stress 216 situations, such as infections and hypoxia, both in term and preterm pregnancies [4, 5, 21]. High 217 copeptin levels at birth are related to acute adverse neonatal outcomes such as IVH [21].

219 The single most important criterion for diagnosis of birth asphyxia is profound metabolic acidosis $220[14,15]$. Notably, copeptin levels increase along with decreasing umbilical cord blood $\mathrm{pH}$ and $\mathrm{BE}$ 221 during normal birth [4], and they are even higher following birth asphyxia [5]. This means that the 222 dynamics of enhanced copeptin plasma concentrations cover a wide range of levels of acidemia from normal to severely abnormal, i.e. those prevailing in birth asphyxia.

In line with the above considerations and data, we demonstrate here that copeptin levels are highly correlated with both arterial cord blood $\mathrm{pH}$ and $\mathrm{BE}$ (fig. 1). The strong and highly significant dependence of high copeptin levels on negative BE shown presently is of particular interest. In excellent agreement with the above data, copeptin levels turned out to be significantly higher in asphyxiated neonates vs controls in the present study. The sensitivity and specificity parameters in the ROC analysis were not as high as previously shown [5], which is attributable to the present inclusion criteria of the control group based on a 1-minute Apgar score of 4 or higher. In contrast, no difference was seen in S100B and EPO levels (fig. 2), most likely because of the relatively mild asphyxia criteria in the present work.

235 A further important finding in the present study is that copeptin levels increased as a function of the 236 total duration of labor and on the duration of the second stage of labor, while EPO and S100B levels did not correlate with either variable. This result with copeptin is most likely explained by very recent observations that just a few contractions (most likely acting via transient periods of minor hypoxia 
on the fetus) are sufficient to trigger detectable AVP/copeptin [24]. Given the cumulative nature of 240 the copeptin levels with a half-life of 30 minutes [25], the above dependence on labor duration is 241 readily explained. Thus, future work on sequential measurements of copeptin may turn out to be valuable in enhancing the prognostic power of this biomarker.

244 It has been previously shown that significantly higher copeptin concentrations are observed after 245 vaginal delivery compared to delivery by primary cesarean section [4]. In the present study, the 246 patient cohort included only neonates born by ECS preceded by periods of labor contractions with 247 variable duration. Thus, it is not surprising that no significant difference in copeptin levels was observed between neonates born vaginally or via ECS. However, copeptin levels were higher after vaginal delivery assisted by vacuum extraction when compared to copeptin levels after ECS or normal vaginal delivery, as reported before [4].

252 To summarize, our study indicates that copeptin has a high potential to become a routine biomarker 253 for neonatal distress and asphyxia. From a (patho)physiological point of view, its advantages are 254 based on the key role of AVP in the adaptations of the fetus to birth. From a practical point of view, 255 it is of much importance that serum copeptin is widely used as a biomarker in adults in emergency 256 departments [13], and therefore this approach can be readily extended to neonatal intensive care units. 257 Future studies are needed to determine whether copeptin concentrations at birth correlate with the 258 severity of HIE, and, more importantly, with long-term neurological outcome following birth 259 asphyxia.

\section{Acknowledgements}

262 The funding of work and materials for blood analyses was provided by Jane and Aatos Erkko 263 Foundation (KK), Sigrid Juselius Foundation (KK) and the Academy of Finland (KK). 
2661 Lee AC, Kozuki N, Blencowe H, Vos T, Bahalim A, Darmstadt GL, Niermeyer S, Ellis M, 267 Robertson NJ, Cousens S, Lawn JE: Intrapartum-related neonatal encephalopathy incidence and 268 impairment at regional and global levels for 2010 with trends from 1990. Pediatr Res 2013;74 269 Suppl 1:50-72.

2702 Ahearne CE, Boylan GB, Murray DM: Short and long term prognosis in perinatal asphyxia: An 271 update. World J Clin Pediatr 2016;5:67-74.

2723 Dalili H, Nili F, Sheikh M, Hardani AK, Shariat M, Nayeri F: Comparison of the four proposed 273 Apgar scoring systems in the assessment of birth asphyxia and adverse early neurologic outcomes. $274 \quad$ PLoS One 2015;10:e0122116.

2754 Wellmann S, Benzing J, Cippa G, Admaty D, Creutzfeldt R, Mieth RA, Beinder E, Lapaire O, 276 Morgenthaler NG, Haagen U, Szinnai G, Buhrer C, Bucher HU: High copeptin concentrations in 277 umbilical cord blood after vaginal delivery and birth acidosis. J Clin Endocrinol Metab $278 \quad 2010 ; 95: 5091-5096$.

2795 Schlapbach LJ, Frey S, Bigler S, Manh-Nhi C, Aebi C, Nelle M, Nuoffer JM: Copeptin 280 concentration in cord blood in infants with early-onset sepsis, chorioamnionitis and perinatal 281 asphyxia. BMC Pediatr 2011;11:38-2431-11-38.

2826 Qian J, Zhou D, Wang YW: Umbilical artery blood S100beta protein: a tool for the early 283 identification of neonatal hypoxic-ischemic encephalopathy. Eur J Pediatr 2009;168:71-77. 
2847 Teramo KA, Widness JA: Increased fetal plasma and amniotic fluid erythropoietin

285 concentrations: markers of intrauterine hypoxia. Neonatology 2009;95:105-116.

2868 Ruth V, Autti-Ramo I, Granstrom ML, Korkman M, Raivio KO: Prediction of perinatal brain 287 damage by cord plasma vasopressin, erythropoietin, and hypoxanthine values. J Pediatr $288 \quad 1988 ; 113: 880-885$.

2899 Beharier O, Kahn J, Shusterman E, Sheiner E: S100B - a potential biomarker for early detection 290 of neonatal brain damage following asphyxia. J Matern Fetal Neonatal Med 2012;25:1523-1528.

29110 Lagercrantz H: Sympathoadrenal Mechanisms during Development; in Polin RA (ed): Fetal and 292 Neonatal Physiology. Saunders, WW. Fox, 1998, pp 220-225.

29311 Evers KS, Wellman S: Arginine Vasopressin and Copeptin in Perinatology. Front Pediatr $2942016 ; 4$

29512 Morgenthaler NG, Struck J, Alonso C, Bergmann A: Assay for the measurement of copeptin, a 296 stable peptide derived from the precursor of vasopressin. Clin Chem 2006;52:112-119.

29713 Nickel CH, Bingisser R, Morgenthaler NG: The role of copeptin as a diagnostic and prognostic 298 biomarker for risk stratification in the emergency department. BMC Med 2012;10:7-7015-10-7.

29914 Yeh P, Emary K, Impey L: The relationship between umbilical cord arterial $\mathrm{pH}$ and serious 300 adverse neonatal outcome: analysis of 51,519 consecutive validated samples. BJOG 2012;119:824301831.

30215 Low AJ: Intrapartum fetal asphyxia: definition, diagnosis, and classification. Am J Obstet 303 Gynecol 1997;176:957-959.

11 
30416 Pihkala J, Hakala T, Voutilainen P, Raivio K: Characteristics of recent fetal growth curves in 305 Finland. Duodecim 1989;105:1540-1546.

30617 Chard T, Hudson CN, Edwards CR, Boyd NR: Release of oxytocin and vasopressin by the 307 human foetus during labour. Nature 1971;234:352-354.

30818 Liu F, Yang S, Du Z, Guo Z: Dynamic changes of cerebral-specific proteins in full-term 309 newborns with hypoxic-ischemic encephalopathy. Cell Biochem Biophys 2013;66:389-396.

31019 Gazzolo D, Di Iorio R, Marinoni E, Masetti P, Serra G, Giovannini L, Michetti F: S100B protein 311 is increased in asphyxiated term infants developing intraventricular hemorrhage. Crit Care Med $312 \quad 2002 ; 30: 1356-1360$.

31320 Seikku L, Rahkonen L, Tikkanen M, Hamalainen E, Rahkonen P, Andersson S, Teramo K, 314 Paavonen J, Stefanovic V: Amniotic fluid erythropoietin and neonatal outcome in pregnancies 315 complicated by intrauterine growth restriction before 34 gestational weeks. Acta Obstet Gynecol 316 Scand 2015;94:288-294.

31721 Benzing J, Wellmann S, Achini F, Letzner J, Burkhardt T, Beinder E, Morgenthaler NG, Haagen 318 U, Bucher HU, Buhrer C, Lapaire O, Szinnai G: Plasma copeptin in preterm infants: a highly 319 sensitive marker of fetal and neonatal stress. J Clin Endocrinol Metab 2011;96:E982-5.

32022 Treschan TA, Peters J: The vasopressin system: physiology and clinical strategies.

321 Anesthesiology 2006;105:599-612; quiz 639-40.

32223 Morgenthaler NG, Struck J, Jochberger S, Dunser MW: Copeptin: clinical use of a new 323 biomarker. Trends Endocrinol Metab 2008;19:43-49. 
32424 Wellmann S, Koslowski A, Spanaus K, Zimmermann R, Burkhardt T: Fetal release of copeptin

325 in response to maternal oxytocin administration: a randomized controlled trial. Obstet Gynecol

326 DOI: 10.1097/AOG.0000000000001594.

32725 L'Abate P, Wiegert S, Struck J, Wellmann S, Cannizzaro V: Determinants of plasma copeptin: a 328 systematic investigation in a pediatric mechanical ventilation model. Respir Physiol Neurobiol $329 \quad 2013 ; 185: 222-227$. 
332 Fig. 1 Dependence of copeptin (a, d), S100B (b, e) and EPO levels (c, f) on pH (a-c) and base excess 333 (BE; d-f). P - and $\mathrm{r}$ - values for each pair are shown in the respective panels, regression lines were 334 drawn when the correlation was significant.

336 Fig. 2 Comparisons between the birth asphyxia and control groups. There were no significant 337 differences in S100B (a) or EPO (b) levels between the groups. Copeptin (c) levels were significantly 338 higher in the asphyxia group compared to the control group. The medians and p-values of Mann339 Whitney U-test are shown (a-c). (d) ROC-curve for cord serum copeptin concentrations in relation to 340 birth asphyxia. The dotted lines indicate the optimal discriminative cut-off of $1522 \mathrm{pmol} / \mathrm{l}$, resulting 341 in a sensitivity of $77 \%$ and a specificity of $70 \%$.

343 Fig. 3 Comparisons between biomarkers and delivery mode. Copeptin (a) levels were significantly 344 higher in neonates born via vacuum extraction compared to emergency cesarean section, whereas no 345 differences in S100B (b) levels were found between groups. EPO (c) levels were significantly higher 346 in neonates born via emergency cesarean section compared to the two other groups. The medians are 347 shown in each panel, and p - values of Kruskal-Wallis test are shown when the difference was 348 significant. 
Table 1. Maternal and fetal/delivery characteristics of the study groups

\begin{tabular}{|c|c|c|c|c|c|}
\hline \multirow[b]{2}{*}{ Maternal characteristics } & \multicolumn{2}{|c|}{ Asphyxia $(n=27)$} & \multicolumn{2}{|c|}{ Control $(n=113)$} & \multirow[t]{2}{*}{$\mathrm{p}$} \\
\hline & & & & & \\
\hline Maternal age, years (mean) & 30.9 & $S D(5.9)$ & 31.1 & $S D(5.5)$ & 0.815 \\
\hline Primiparity & 15 & $(55.6)$ & 62 & $(54.9)$ & 1.000 \\
\hline In vitro fertilization & 2 & $(7.4)$ & 5 & $(4.4)$ & 0.620 \\
\hline Smoking & 4 & $(14.8)$ & 9 & $(8.0)$ & 0.277 \\
\hline Obesity (body mass index $\geq 30 \mathrm{~kg} / \mathrm{m}^{2}$ ) & 2 & $(7.4)$ & 17 & $(15.0)$ & 0.530 \\
\hline Gestational diabetes & 4 & $(14.8)$ & 14 & $(12.4)$ & 0.751 \\
\hline Chronic hypertension & 1 & $(3.7)$ & 3 & $(2.7)$ & 0.580 \\
\hline \multicolumn{6}{|l|}{ Fetal/delivery characteristics } \\
\hline Spontaneous vaginal delivery & 7 & $(25.9)$ & 49 & $(43.4)$ & 0.257 \\
\hline Vacuum extraction & 9 & $(33.3)$ & 24 & $(21.2)$ & 0.309 \\
\hline Emergency cesarean section & 11 & $(40.7)$ & 40 & (35.4) & 0.727 \\
\hline Gestational weeks at birth & 41.1 & $(40.0-41.6)$ & 40.7 & $(39.7-41.7)$ & 0.372 \\
\hline Post-term births $\left(\geq \mathrm{H} 42^{+0}\right)$ & 5 & $(18.5)$ & 22 & $(19.5)$ & 1.000 \\
\hline Male & 17 & $(63.0)$ & 66 & $(58.4)$ & 0.828 \\
\hline Birth weight (g) & 3520 & $(3260-3945)$ & 3656 & $(3282-3991)$ & 0.499 \\
\hline Relative birth weight (SD) & -0.13 & $(-1.02-0.62)$ & 0.02 & $(-0.67-0.81)$ & 0.385 \\
\hline 5 min Apgar score & 6 & $(4-7)$ & 8 & $(6-9)$ & $<0.0001$ \\
\hline 10 min Apgar score & 8 & $(6-9)$ & 9 & $(8-9.5)$ & 0.0009 \\
\hline Umbilical artery $\mathrm{pH}$ & 7.03 & $(6.97-7.08)$ & 7.21 & $(7.14-7.30)$ & $<0.0001$ \\
\hline Umbilical artery base excess & -12.7 & $(-14.4--11.1)$ & -5.80 & $(-7.85--2.85)$ & $<0.0001$ \\
\hline Umbilical artery $\mathrm{pO}_{2}(\mathrm{kPa})$ & 1.9 & $(1.1-2.8)$ & 2.4 & $(1.7-3.3)$ & 0.029 \\
\hline Umbilical artery $\mathrm{pCO}_{2}(\mathrm{kPa})$ & 10.1 & $(9.2-12.7)$ & 7.8 & $(6.7-9.2)$ & $<0.0001$ \\
\hline Umbilical serum erythropoietin (U/l) & 71.7 & $(22.2-116.0)$ & 46.1 & $(22.2-124.5)$ & 0.683 \\
\hline Umbilical serum S100B $(\mu \mathrm{g} / \mathrm{l})$ & 0.33 & $(0.19-0.63)$ & 0.31 & $(0.24-0.45)$ & 0.712 \\
\hline Umbilical serum copeptin (pmol/l) & 2279 & $(1476-3144)$ & 973.7 & $(320.9-1961)$ & $<0.0001$ \\
\hline
\end{tabular}

Median (interquartile range) or number (percentage) are shown

Table 2. Biomarker correlations to other variables Copeptin

\section{S100B}

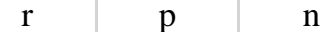

5 min Apgar score

10 min Apgar score

Gestational age

Birth weight

Total labor duration

Second stage labor duration

\begin{tabular}{|l|l|l|l}
$\mathrm{n}$ & $\mathrm{r}$ & $\mathrm{p}$ & \\
\hline
\end{tabular}

\begin{tabular}{|l|c|c|}
\hline 139 & -0.2998 & $0.0004^{*}$
\end{tabular}

\begin{tabular}{|l|l|l|l|}
139 & -0.1678 & $0.0484 *$ & 139 \\
\hline
\end{tabular}

\begin{tabular}{|l|l|l|l|}
139 & 0.0644 & 0.4510 & 139 \\
\hline
\end{tabular}

\begin{tabular}{|l|l|l|l|}
139 & -0.1713 & $0.0438 *$ & 139 \\
\hline
\end{tabular}

\begin{tabular}{|l|l|l|l|}
\hline 88 & 0.3267 & $0.0019 *$ & 89 \\
\hline
\end{tabular}

\begin{tabular}{l|l|l|l|}
88 & 0.2787 & $0.0086 *$ & 89 \\
\hline
\end{tabular}

EPO

\begin{tabular}{c|c|c|c|c|}
$\mathrm{r}$ & $\mathrm{p}$ & $\mathrm{n}$ & $\mathrm{r}$ & $\mathrm{p}$ \\
\hline-0.2041 & $0.0159^{*}$ & 140 & -0.2914 & $0.0005^{*}$ \\
-0.2048 & $0.0156^{*}$ & 140 & -0.1380 & 0.1040 \\
\hline-0.0018 & 0.9836 & 140 & 0.4513 & $<0.0001^{*}$ \\
-0.0235 & 0.7838 & 140 & -0.0367 & 0.6672 \\
\hline 0.1247 & 0.2444 & 89 & 0.0656 & 0.5414 \\
\hline 0.1038 & 0.3332 & 89 & 0.0162 & 0.8802 \\
\hline
\end{tabular}


$359 \quad$ Fig. 1

A

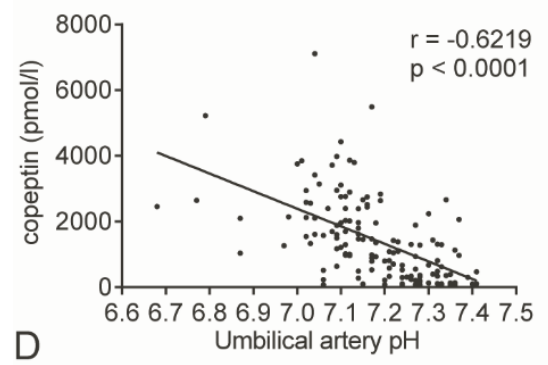

D Umbilical artery $\mathrm{pH}$

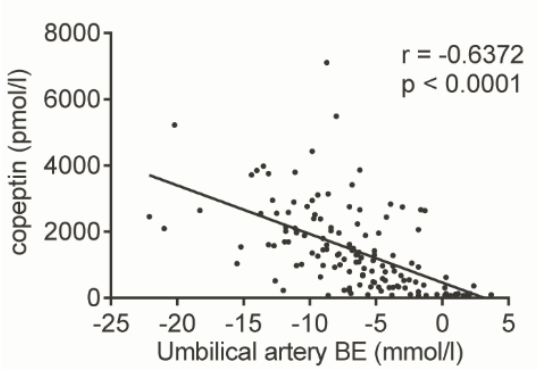

B
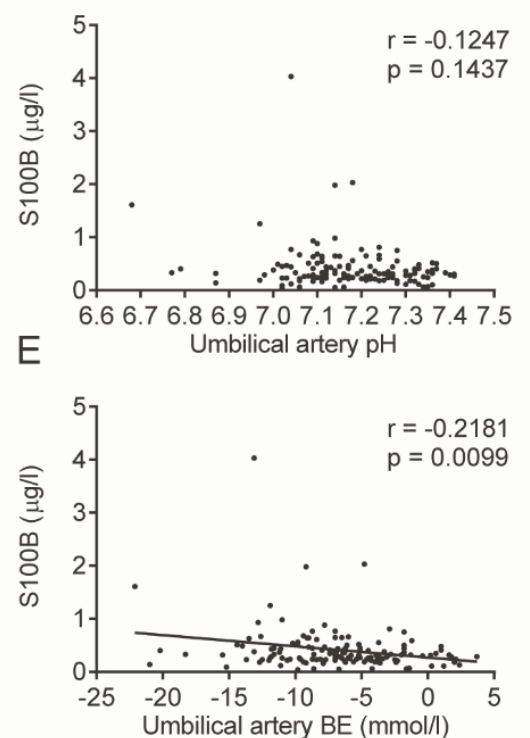

C
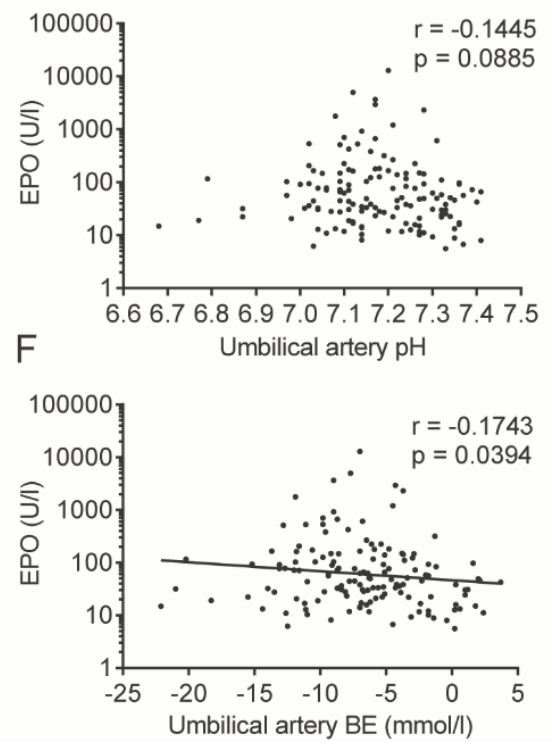

Fig. 2 
A

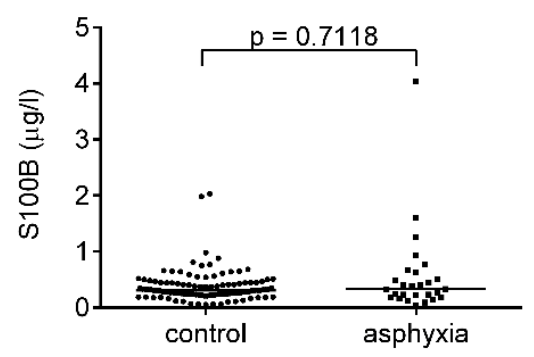

C

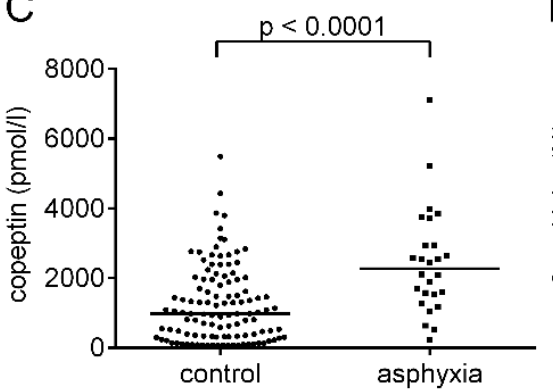

363

364

365 Fig. 3

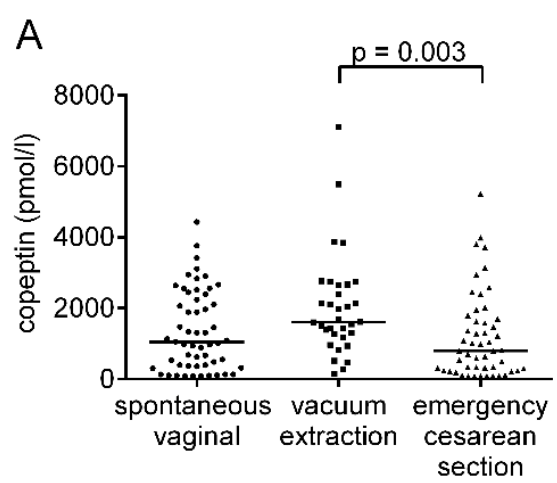

B

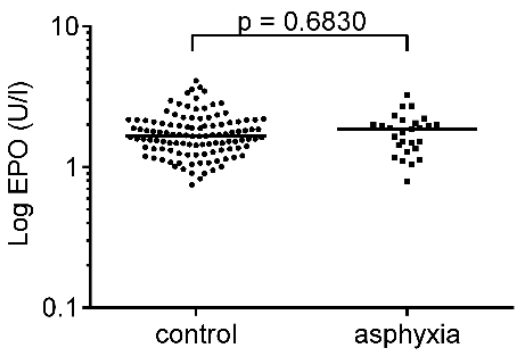

D

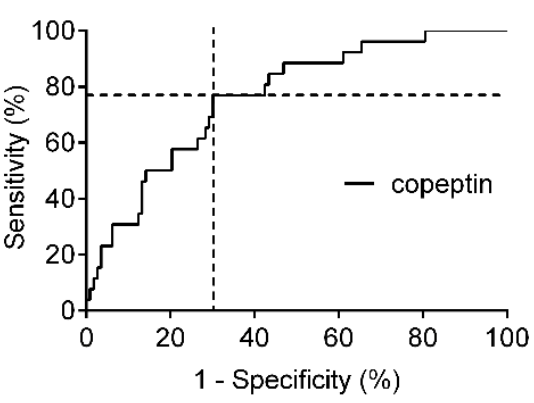

B

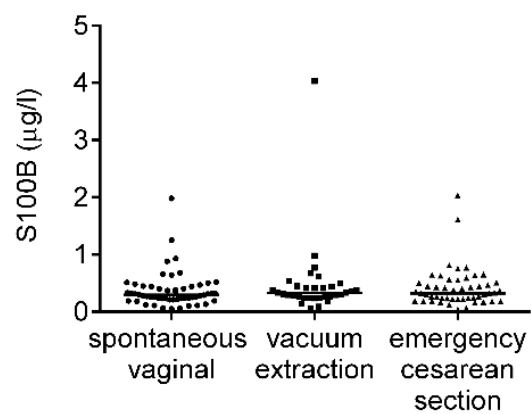

C

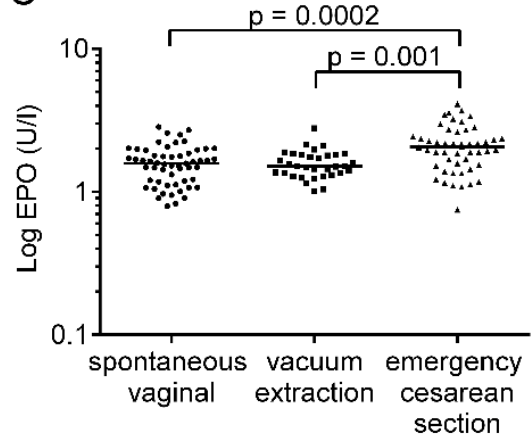

